\title{
COHERENCIA ENTRE PERFILES DE EGRESO E INSTRUMENTOS DE EVALUACIÓN EN CARRERAS DE EDUCACIÓN BÁSICA EN CHILE ${ }^{1}$
}

\author{
Isabel Möller y Héctor Gómez²
}

\begin{abstract}
RESUMEN
El presente artículo expone los resultados del análisis de la relación existente entre las competencias o habilidades que declaran los perfiles de egreso de diferentes carreras de Educación Básica y los indicadores de evaluación utilizados en asignaturas del plan de estudios, con el objetivo de evaluar su correspondencia y gradualidad en la consecución y desarrollo de estas competencias y, por tanto, el grado de coherencia entre perfiles y evaluaciones, específicamente en las asignaturas de Evaluación, Currículum y Didáctica de cuatro instituciones. Las principales conclusiones del estudio señalan que si bien los docentes demuestran poseer una cultura evaluativa que considera el uso de criterios e indicadores sistematizados en pautas conocidas por los estudiantes, no existen estrategias que aseguren el logro progresivo de las habilidades declaradas en el perfil como un ejercicio consciente e intencionado de incorporación de las competencias a cada asignatura y a sus respectivos instrumentos de evaluación, lo que da como resultado que muchas veces partes importantes de aquellas, quedan sin ser evaluadas.
\end{abstract}

Palabras clave: competencias, habilidades, indicadores de evaluación, plan de estudios, educación primaria.

\section{COHERENCY BETWEEN GRADUATE PROFILES AND ASSESSMENT INSTRUMENTS IN PRIMARY EDUCATION TEACHER PREPARATION PROGRAMS IN CHILE}

\section{ABSTRACT}

The present article presents the results of an analysis of the existing relationship between the competencies or skills, declared in the graduate profiles of different primary education teacher preparation programs, and the evaluation indicators used in the various disciplines outlined in the curriculum. The study aims to evaluate the correspondence and progression in the attainment and development of said competencies and in turn, the degree of consistency between the profiles and assessments, specifically for the disciplines of Assessment, Curriculum and Instruction, of four institutions of higher learning. The main findings of the study indicate that although the teachers demonstrate a culture of assessment, which considers the use of systematized criteria and indicators in assessment frameworks familiar to the students, there are no strategies, by way of an intentional and reflective practice of incorporating the competencies of each discipline and their respective assessment tools, that would guarantee the progressive attainment of the skills declared in the profiles. As a result, significant aspects of the graduate profiles remain unassessed.

Keywords: competencies, skills, evaluation indicators, curriculum, primary education

1 El presente proyecto recibió apoyo de la Convocatoria de apoyo a la investigación en educación del Consejo Nacional de Educación 2013.

2 Facultad de Educación, Universidad Católica Silva Henríquez, Santiago, Chile. Contactos: isabelmollerl@gmail.com y hrgomez@uc.cl 


\section{Introducción}

La presente investigación se propone analizar la relación existente entre las competencias o habilidades que declaran los perfiles de egreso de diferentes carreras de Pedagogía en Educación Básica y los indicadores de evaluación utilizados en los instrumentos de corrección de diferentes asignaturas, a lo largo del plan de estudios de estas carreras. Ello, con el objetivo de evaluar su correspondencia y progresión en la consecución y desarrollo de estas competencias, específicamente, en las asignaturas de Evaluación, Currículum y Didáctica.

De este modo, los objetivos específicos de la investigación apuntan a:

- identificar los perfiles de egreso de las carreras y el escalamiento de las habilidades y/o competencias dentro de los planes de estudio;

- identificar los instrumentos de evaluación y corrección de las asignaturas mencionadas;

- recabar información acerca de la percepción de los alumnos y profesores respecto de las evaluaciones que desarrollan y su relación con las competencias o habilidades descritas en el perfil de egreso;

- comparar y contrastar la documentación acerca de los instrumentos de evaluación y corrección de cada asignatura y las competencias o habilidades declaradas y vinculadas con ellas; y finalmente,

- desarrollar un diagnóstico y propuestas de mejora respecto de la coherencia entre los respectivos perfiles e indicadores de evaluación en el contexto del estudio.

De esta forma, la hipótesis del presente estudio sostiene que los indicadores de evaluación de las asignaturas seleccionadas, aún no serían congruentes con los perfiles de egreso declarados en las carreras, existiendo una cultura evaluativa en la que aún el perfil de egreso no es un factor a considerar al momento de diseñar los instrumentos de evaluación y corrección. 


\title{
1. Antecedentes
}

Frente a los resultados que arrojó la prueba Inicia durante 2011 y 2012, debiésemos preguntarnos por qué la mayor parte de los egresados de Pedagogía Básica que rindieron el examen obtuvieron resultados, al menos, preocupantes en el cumplimiento de los estándares orientadores para egresados de Pedagogía (en varios casos resultados menores al 50\% de logro), si su solo egreso implica que obtuvieron una calificación "suficiente" en todos los cursos impartidos por el programa curricular. A pesar de que todos quisiéramos instrumentos de diagnóstico más completos y holísticos que esta prueba, pareciera ser a primera vista que la evaluación en la educación superior no está siendo congruente con los nuevos modelos educativos que basan su enseñanza en perfiles de egreso y en estándares orientadores. La importancia de la evaluación en educación hace ya varias décadas que no se cuestiona, sin embargo, es su función la que debiese cambiar, bajo un modelo por competencias o por habilidades, así como lo señalan Jorba y Sanmartí (2008b):

\begin{abstract}
“(...) cada vez más se considera que si se quiere cambiar la práctica educativa es necesario cambiar la práctica evaluativa, es decir, su finalidad y el qué y cómo se evalúa (...). Se puede decir que la evaluación pone al descubierto parte del llamado currículum oculto del profesorado. Planteamientos didácticos aparentemente innovadores pueden ser discutidos cuando se observa qué y cómo se evalúan los aprendizajes promovidos" (p. 21).
\end{abstract}

Es decir, el fin y la forma de la evaluación aún no son congruentes con los perfiles de egreso declarados en las carreras, en nuestro caso Pedagogía Básica, permaneciendo estos aún en el plano declarativo y no en lo concreto y evidenciable, como por ejemplo, en los planes de evaluación o en los indicadores de evaluación de los instrumentos de corrección. Los autores recién citados proponen que la evaluación amplíe su función meramente acreditadora o seleccionadora y reconozca dos funciones amplias:

“(...) una de carácter social de selección y de clasificación, pero también de orientación del alumnado y una función de carácter pedagógico, de regulación del proceso de enseñanza- 
aprendizaje, es decir, de reconocimiento de los cambios que se han de introducir progresivamente en este proceso para que todos los alumnos aprendan de forma significativa" (p. 22).

En este sentido, la presente investigación no asume como incuestionables o como única guía los estándares orientadores para egresados de Pedagogía que emanan del Ministerio de Educación, ni las pruebas estandarizadas que intentan medir la capacidad de un egresado para ejercer la docencia. Del mismo modo, trata acerca de la capacidad que tienen los equipos docentes de poner efectivamente en práctica un proyecto de formación profesional pedagógica y de llevarlo a las prácticas evaluativas en sus asignaturas. Todo ello sin juzgar si el proyecto educativo considera como fuente principal los estándares nacionales, o un proyecto propio, o referentes de organizaciones ciudadanas, o referentes internacionales. No poseemos tampoco un estudio acabado de las fuentes que han predominado en la definición de perfiles de egreso, ni de los mecanismos, democráticos o no, para diseñarlos, de las universidades participantes ni para todas las universidades a nivel nacional.

No obstante, Struyven, Dochy y Janssens (2005) han estudiado la crucial influencia que la evaluación tiene sobre la manera en que estudian y aprenden los alumnos, lo cual reafirma estudios realizados la década de los noventa (Entwistle, 1991; Marton \& Säljö, 1997). Como señala lapidariamente Boud (1995): "los estudiantes pueden, con dificultad, escapar de los efectos de una pobre enseñanza, pero no pueden escapar (por definición, si quieren licenciarse) de los efectos de una mala evaluación" (p. 35). Es en este sentido que el aprendizaje está más vinculado con las prácticas evaluativas que con las prácticas de enseñanza. Más incisiva es en este sentido María Antonieta Casanova (2007), quien señala;

"Los alumnos estudian para aprobar. Los profesores enseñan para que sus alumnos superen las evaluaciones. Lo que tiene valor real en la enseñanza es lo que se evalúa; de lo contrario, nadie lo tiene en cuenta. Las familias se preocupan de la situación de aprendizaje de sus hijos cuando estos suspenden" (p. 59-60). 
Sin embargo, a pesar de que los docentes saben que la mayor parte de sus alumnos orienta sus aprendizajes en función de las evaluaciones que se les aplicarán (pues son ellas las que determinan la aprobación o reprobación del curso), no se ha realizado un trabajo de coordinación concreta, a nivel de la educación superior, entre la enseñanza por competencias o resultados de aprendizaje y la evaluación-aprendizaje por competencias o resultados de aprendizaje.

\section{Marco conceptual}

La presente investigación pretende situarse dentro del paradigma constructivista, a la vez que bajo la concepción de la enseñanzaaprendizaje y de la evaluación por competencias (modelo educativo por competencias). Desde este campo de estudio repasaremos conceptos como la formación inicial docente, las competencias, el modelo curricular por competencias en la educación superior y formación docente, perfil de egreso y la evaluación por competencias.

\subsection{Formación inicial docente}

Tradicionalmente se ha entendido a la formación inicial docente como un proceso de formación para desempeñarse en el mundo de la educación, que incorpora un conjunto de contenidos, habilidades y disposiciones entregadas durante la formación académica y sus distintas orientaciones, extensiones y problemáticas.

Esta reflexión deviene de los aportes de una serie de autores como Mercado (2010), quien señala que la formación de maestros debe ser entendida como un proceso continuo, que no debe parcelarse en etapas iniciales, prácticas y posteriores, por lo cual es relevante hablar de procesos permanentes y continuos.

Asimismo Perrenoud (2001) afirma que este proceso, más que un concepto neutro, debe ser entendido como concepto socialmente construido, que varía en el marco de las relaciones y condiciones sociales e históricas, requiriendo como componente básico la formación humana integral, una sólida preparación en disciplinas y competencias relacionadas con el desarrollo del aprendizaje y la 
introducción a la práctica de la enseñanza, bajo la guía de un maestro experimentado.

A su vez, Vaillant (2004) complementa lo anterior destacando que este campo representa "uno de los problemas más difíciles enfrentados por los gobiernos y las sociedades por sus implicancias políticas, ideológicas y financieras" (p. 6). Esta complejidad a la que alude la autora, expresa la fragilidad y complejidad del tratamiento de la formación de profesores, en cuanto a la participación de múltiples discursos en juego, los límites de la formación, la diversidad de contextos en donde se desarrolla y el nivel de responsabilidad asignado a este ámbito en el contexto global de la educación y calidad de la formación.

Lo anterior, queda en evidencia a través de la creciente preocupación académica e investigativa a nivel nacional e internacional en este campo. Prueba de ello han sido las investigaciones como Teacher's Matter (OCDE, 2005) y el Informe Mackinsey (Barber $\&$ Mourshed, 2008), que ponen al centro del debate la calidad en educación y su relación con la formación docente.

De este modo, tanto Europa como Estados Unidos han ido progresivamente instalando estándares mínimos, evaluaciones del desempeño, procesos de selección al ingreso, prácticas profesionales progresivas, cambios curriculares, reformas políticas y una serie de transformaciones que apuntan a profesionalizar la labor docente y, en conjunto, elevar la calidad de la educación y los aprendizajes de los estudiantes (Gómez, 2013).

En América Latina las experiencias han sido muchas, pero las variaciones entre ellas no han sido significativas, necesitándose a juicio de Vaillant (2009) un cambio de enfoque, en tanto las reformas implementadas en los años noventa en la región habrían tenido un escaso impacto en las prácticas, las cuales siguieron obedeciendo a viejos modelos.

De este modo, en la actualidad se vive un proceso de absorción de las políticas internacionales en torno a la formación docente, lo cual a juicio de Cardelli y Duhalde (2001) converge en la globalización de 
la cultura y la homogeneización de los discursos, como un proceso de transformaciones derivado de la mercantilización de los objetos de la cultura y en particular del conocimiento científico y técnico; de las acciones y discursos que dan nuevo impulso a la demanda educativa, generando una centralidad y uniformidad de la formación inicial docente.

En nuestro país, Ávalos (2009) señala que la formación docente corresponde a "un sistema grande y fragmentado de carreras de Pedagogía, carente de regulación externa y sobre el que existen dudas respecto de la capacidad docente de muchos de sus egresados" (p. 276).

Este crudo diagnóstico ha contribuido decididamente a generar diversas iniciativas de cambio, que se han orientado hacia una renovación de los procesos de formación docente, producto entre otros aspectos de una:

"(...) débil articulación entre la formación pedagógica y la formación de especialidad; (...) el nivel de aprendizaje de los contenidos curriculares y la comprensión de la naturaleza teórico-práctica de la acción pedagógica resultan insuficientes; que las relaciones con el sistema educativo son aún incipientes y que pese a las mediciones externas (como la prueba Inicia), no se ha avanzado en procedimientos internos confiables de monitoreo y evaluación a los programas de formación" (Cisternas, 2011, p. 141).

De este modo, una de las alternativas ha sido optar por un proceso de renovación curricular basado en el modelo de competencias profesionales, que a continuación pasamos a abordar.

\subsection{Competencias profesionales en la formación docente}

Existen una serie de definiciones del concepto de "competencia", tanto de instituciones y programas, como de investigadores y equipos de investigación. Sin embargo, una de las definiciones más utilizadas en educación, por su carácter integrador, es la que nos entrega Perrenoud (2001), ya que permite abordar el concepto no solo desde el ámbito laboral, sino también humano, pues contiene en ella la noción de movilización de recursos, tanto personales como especializados, para 
realizar así una aplicación y relación entre los mismos, contextualizada en situaciones no solo laborales, sino de todo tipo.

Perrenoud define la competencia como una capacidad de actuar de manera más eficaz en un tipo definido de situación y que se apoya en conocimientos, aunque no se reduce a ellos. La competencia es para él una "aptitud para enfrentar eficazmente una familia de situaciones análogas, movilizando a conciencia y rápida, pertinente y creativamente, múltiples recursos cognitivos: saberes, capacidades, microcompetencias, informaciones, valores, actitudes, esquemas de percepción, de evaluación y de razonamiento" (Perrenoud, 2004). Como lo señalara Delors (1995), la competencia implica dominar el "saber", el "saber hacer", "saber convivir" y el "saber ser".

De este modo, en el ámbito de la formación universitaria, la idea de competencia posee un carácter integrador (Pavié, 2011a), en tanto su definición surge de las características de un determinado perfil profesional, de las demandas de la actividad futura a ser realizada por el profesional en su respectivo campo, así como de los propósitos formativos propios de la institución como parte del título que busca otorgar.

Si bien existe y seguirá existiendo preocupación acerca de la implementación de un currículo basado en competencias en el campo de la formación docente, el centro de preocupación del diseño de este tipo es asegurar que los y las estudiantes serán capaces de demostrar sus capacidades aprendidas después de que hayan adquirido una combinación de conocimientos, habilidades y destrezas, entendidos integralmente como desempeños.

El desafío, sin embargo, está en poder evaluar de forma coherente lo establecido en el currículo, los respectivos planes de estudio, actividades curriculares y sus programas, con los desempeños específicos y observables, o como plantean Knust y Gómez (2009) "cómo crear un balance entre aprendizaje y desarrollo de competencias en concordancia con los sistemas de evaluación que sea coherente con los resultados que se espera que demuestren al final de un proceso formativo" (p. 106). 


\subsection{Perfil de egreso}

Los modelos de formación por competencias o por resultados de aprendizaje, de acuerdo con la elección de cada institución y/o programa, han intentado operacionalizarse en las distintas instituciones a través de la definición de perfiles de egreso.

A partir del proceso de renovación curricular que se está llevando a cabo en las instituciones de educación superior a nivel nacional, las universidades están desarrollando serios esfuerzos por definir qué entienden por perfil de egreso. Un ejemplo de ello lo encontramos dentro del Manual para la implementación del sistema de créditos transferibles de la Universidad de Santiago de Chile (Usach, 2013), en el cual se señala que "el perfil de egreso establece las áreas del conocimiento, habilidades y destrezas profesionales, actitudes, valores y competencias que desarrolla el plan de estudios" (p. 43). De este modo, el perfil de egreso no se aboca solo al ámbito laboral, sino que implica una definición integral del profesional que se espera formar. Asimismo, Hawes (2010) concibe el perfil de egreso como la declaración formal que hace la institución frente a la sociedad y frente a sí misma, comprometiendo la formación de una identidad profesional, ciertos compromisos formativos en ámbitos de realización de la profesión y sus competencias clave asociadas.

Así entendido el perfil de egreso (como definición identitaria y como compromiso formativo), se concibe como un instrumento que dota de sentido a los programas de formación, por lo que resulta fundamental que los perfiles incluyan mecanismos para evaluar su cumplimiento.

\subsection{Evaluación de competencias profesionales}

Con relación al concepto de evaluación, Hagar, Athanasou y Gonczi (1994) la definen como un proceso de recolección de evidencias y de formulación de juicios acerca de la medida y la naturaleza del progreso hacia los desempeños requeridos, establecidos en un estándar o un resultado del aprendizaje. En la misma línea, Ramírez y Santander (2003) conciben la evaluación como el proceso de recolección de datos del aprendizaje, el cual se asocia con el uso de los instrumentos 
de evaluación y se entremezcla con instrumentos e indicadores de evaluación que pretenden eventualmente diagnosticar cuánto ha aprendido el alumno.

El presente estudio trabajará sobre la base del concepto de evaluación que nos entrega Casanova (2007), especialmente por el sentido que le otorga al proceso evaluativo:

"La evaluación aplicada a la enseñanza y el aprendizaje consiste en un proceso sistemático y riguroso de recogida de datos, incorporado al proceso educativo desde su comienzo, de manera que sea posible disponer de información continua y significativa para conocer la situación, formar juicios de valor con respecto a ella y tomar las decisiones adecuadas para proseguir la actividad educativa, mejorándola progresivamente" (p. 60).

Coincidiendo con el propósito de la evaluación, Ahumada (2006) señala como centro del proceso evaluativo al aprendizaje:

"El elemento esencial de un proceso evaluativo centrado en el aprendizaje debe apuntar a establecer niveles de avance o progreso en el acercamiento a un determinado conocimiento, tomando en consideración su importancia significativa o su relación con los conocimientos previos que posee cada estudiante" (p. 30).

Coincidiendo con Ahumada (2006), McDonald, Boud; Francis $\&$ Gonczi (2000) recalcan que el uso tradicional que se le ha dado a la evaluación ha terminado convirtiéndose en su finalidad, a pesar de que este no sea su sentido:

“(...) frecuentemente, la evaluación responde a las necesidades del juicio sumativo, no del aprendizaje y se concentra más en el registro y la medición que en la comunicación y descripción de la competencia lograda. Irónicamente, a menudo tampoco sirve muy bien a las necesidades de lo primero" (p. 47).

Respecto de la evaluación por competencias, los mismos autores señalan en primer lugar que esta presenta múltiples desafíos:

"(...) la competencia no puede ser observada directamente, pero puede ser inferida del desempeño. Por ello es que se requiere 
pensar acerca de los tipos de desempeños que permitirán reunir evidencia, en cantidad y calidad suficiente, para hacer juicios razonables acerca de la competencia de un individuo" (p. 54).

En nuestro país, Hawes (2005) señala que los temas de evaluación "están entre los que más problemas ponen a quienes ejercen la docencia" (p. 7), específicamente en el ámbito universitario, donde no necesariamente los docentes poseen la formación especializada requerida en cuestiones evaluativas y, en específico, en la evaluación por competencias.

De este mismo modo, Salas (2009) enuncia las implicancias de la evaluación por competencias en el ámbito de la formación docente, debiendo pasar de una evaluación por logros a una de procesos, lo que conlleva un seguimiento al proceso de aprendizaje desde la motivación misma hasta la ejecución de la acción y su consecuente resultado.

Por último McDonald, Boud, Francis y Gonczi (2000) sintetizan el sentido de la evaluación por competencias en la educación superior de la siguiente forma:

¿Por qué la evaluación basada en competencias? Un enfoque de evaluación basado en competencias es altamente apropiado para la formación profesional:

- para asegurar que la enseñanza y la evaluación estén al servicio de los resultados requeridos (en lugar de basar la enseñanza y la evaluación, por ejemplo, en los cursos realizados o en el tiempo utilizado);

- para facilitar el otorgamiento de créditos por la competencia adquirida en otros lugares;

- para ayudar a los estudiantes a comprender claramente lo que se espera de ellos si quieren tener éxito en el curso; y

- para informar a los empleadores potenciales qué significa una calificación particular.

Este último punto es muy importante en el caso de las carreras de Pedagogía que deben acreditar que sus egresados poseen todas las competencias requeridas para poder formar y educar a sus futuros alumnos. 


\section{Metodología}

El enfoque de esta investigación se desarrolla en un marco cualitativo, perspectiva metodológica que pretende interpretar el contexto por medio de técnicas e instrumentos, los cuales se aplican en el mismo escenario en el que se encuentran los sujetos y los objetos de estudio: docentes, estudiantes, perfiles, programas de las actividades curriculares, planes de evaluación e instrumentos de evaluación, interpretando la realidad según la construcción y significación que realizan los propios actores involucrados (Denzin \& Lincoln, 2012).

Asimismo, es de tipo exploratoria y pretende operacionalizarse a nivel descriptivo (pues pretende diagnosticar), evaluativo (pues pretende emitir un juicio acerca de la correspondencia entre dos aspectos que debiesen ser coherentes) y propositivo (pues pretende sugerir propuestas de mejora, sin necesariamente ejecutarlas directamente, sino poniendo a disposición de los ejecutores de los cursos las propuestas).

Del mismo modo, propone un diseño de estudios de caso basado en la correspondencia entre los indicadores de evaluación y los resultados de aprendizaje o competencias declaradas en los perfiles de egreso para tres asignaturas de cada una de las carreras de Pedagogía Básica, de las cuatro universidades participantes.

El diseño de la investigación corresponde a la aplicación de técnicas e instrumentos que contribuyen a la consecución de los objetivos específicos propuestos y, con ello, al objetivo general de investigación.

De este modo, para cada uno de los objetivos se establece una técnica e instrumento específico para la recolección de información, que luego se analiza por medio de matrices de análisis de contenido, según categorías y criterios previamente definidos, en consideración con los casos, sujetos y fuentes documentales.

Finalmente, la investigación considera el levantamiento de sugerencias de mejora en pro de la coherencia entre las competencias declaradas en los perfiles de egreso de las carreras de Pedagogía en 
Educación Básica seleccionadas y los indicadores de evaluación utilizados en los instrumentos de corrección.

Lo anterior, se clarifica en la siguiente tabla:

Tabla 1: Diseño metodológico

\begin{tabular}{|c|c|c|c|c|}
\hline $\begin{array}{l}\text { Objetivo } \\
\text { específico }\end{array}$ & Técnica & $\begin{array}{l}\text { Instrumento de recogida } \\
\text { de información }\end{array}$ & $\begin{array}{l}\text { Instrumento de análisis } \\
\text { de información }\end{array}$ & $\begin{array}{l}\text { Tipo de } \\
\text { análisis }\end{array}$ \\
\hline \multirow{2}{*}{1} & ENTREVISTAS & $\begin{array}{l}\text { Guion de entrevista } \\
\text { semiestructurada }\end{array}$ & $\begin{array}{l}\text { Matriz de análisis de } \\
\text { entrevista a docentes }\end{array}$ & \multirow{4}{*}{$\begin{array}{l}\text { ANÁLISIS DE } \\
\text { CONTENIDO }\end{array}$} \\
\hline & FOCUS GROUP & Guion de focus group & $\begin{array}{l}\text { Matriz de análisis de } \\
\text { focus group a estudiantes }\end{array}$ & \\
\hline 2 & $\begin{array}{l}\text { ANÁLISIS } \\
\text { DOCUMENTAL }\end{array}$ & $\begin{array}{l}\text { Matriz de análisis } \\
\text { Instrumentos de } \\
\text { evaluación y corrección y } \\
\text { perfil de egreso }\end{array}$ & $\begin{array}{l}\text { Matriz de análisis } \\
\text { Instrumentos de } \\
\text { evaluación y corrección y } \\
\text { perfil de egreso }\end{array}$ & \\
\hline 3 & $\begin{array}{l}\text { ANÁLISIS } \\
\text { DOCUMENTAL }\end{array}$ & $\begin{array}{l}\text { Matriz de análisis } \\
\text { Instrumentos de } \\
\text { evaluación y corrección y } \\
\text { perfil de egreso }\end{array}$ & $\begin{array}{l}\text { Matriz de análisis } \\
\text { Instrumentos de } \\
\text { evaluación y corrección y } \\
\text { perfil de egreso }\end{array}$ & \\
\hline
\end{tabular}

La muestra corresponde a cuatro casos (universidades), considerando diversos tipos de instituciones. Sin embargo aquí debió predominar el criterio relacionado con la voluntad de participar por parte de la institución. La recolección de información se llevó a cabo entre septiembre de 2013 y julio de 2014.

De este modo, la presente investigación incluye una universidad de carácter confesional-privada (caso 1), laica-estatal (caso 2), confesional/humanista-privada (caso 3) y laica-privada (caso 4).

En una segunda instancia se definió la cantidad y el tipo de asignaturas por diagnosticar. El número de asignaturas por cada universidad se limitó a tres: Evaluación, Currículum y Didáctica (dos en el caso de las universidades que contemplan en una sola asignatura las áreas de currículum y evaluación, como es el caso 4).

En una tercera instancia se identificaron las fuentes documentales que podrían proporcionar información relevante respecto del estudio. Se definieron cuatro fundamentales: 
- planes de estudio y los perfiles de egreso de las carreras de Pedagogía General Básica de las universidades participantes;

- programas de asignatura;

- plan de evaluación, instrumentos y pautas de evaluación de cada una de las asignaturas seleccionadas; y

- entrevistas a los profesores que imparten las asignaturas seleccionadas y grupos focales con estudiantes participantes de las asignaturas seleccionadas.

Respecto de la construcción y validación de instrumentos para la recolección de información, se seleccionaron dos tipos: entrevistas semiestructuradas de respuesta abierta y guion de entrevista semiestructurada de respuesta abierta para los grupos focales. Posteriormente, se diseñaron otros para el análisis de la documentación, los cuales fueron sometidos a juicio de experto.

Una vez recogida la información a partir de los instrumentos señalados, se procedió a realizar el análisis, a través del levantamiento de matrices que consideran categorías y criterios previamente definidos y en coherencia con los instrumentos de investigación. Para efectos analíticos, se estimó adecuado realizar análisis de contenido que como técnica promueve la identificación y explicación de las representaciones sociales que otorgan sentido al relato comunicativo de las interacciones verbales (Bardin, 2002). El análisis documental se complementó con un análisis de frecuencia, en el que se estudia el número de veces que se presenta una variable.

Se realizaron dos tipos de análisis que agrupan a los objetivos específicos: respecto de las percepciones de docentes y de estudiantes en relación con los procesos evaluativos; y acerca de la correspondencia entre perfiles de egreso e indicadores de evaluación para los cuatro casos y las respectivas actividades curriculares seleccionadas.

Respecto del primer análisis y, en específico de los sujetos docentes se consideraron las dimensiones de:

- concepciones de la evaluación;

- coherencia y pertinencia; 
- usos de la evaluación y sus resultados; y

- metacognición de la evaluación.

Todas las dimensiones se desagregaron a su vez en criterios de análisis. En cuanto a los estudiantes, las dimensiones específicas fueron:

- concepciones de la evaluación;

- preparación de las evaluaciones;

- evaluaciones y uso de los resultados;

- metacognición de la evaluación; y

- propuestas.

Del mismo modo que en el caso de los docentes, las dimensiones se desagregaron en criterios de análisis. Los análisis de ambos sujetos se integraron para cada una de las categorías, con el propósito de encontrar claves explicativas para comprender el fenómeno de la coherencia entre las evaluaciones de cada asignatura y el perfil de egreso.

El segundo análisis, se enfocó en la correspondencia entre perfiles de egreso e indicadores de evaluación para los cuatro casos y las respectivas actividades curriculares seleccionadas. En este contexto, el procedimiento consistió en el ingreso de competencias o habilidades del perfil de egreso relacionadas con cada asignatura de manera explícita y/o implícita, los objetivos o resultados de aprendizaje del programa de cada asignatura y sus correspondientes instrumentos de corrección, a una matriz de análisis con dimensiones predefinidas. Las dimensiones de análisis establecidas fueron: relación entre el plan de evaluaciones de la asignatura, con los objetivos, tipo, descripción general y pauta de evaluación de cada instancia de evaluación sumativa; y correspondencia entre los indicadores de evaluación y las competencias o resultados de aprendizaje del perfil de egreso asociadas a la asignatura.

Finalmente se construyeron sugerencias generales de mejora de correspondencia basadas en los resultados emanados del análisis I y II. 


\section{Análisis y resultados}

De acuerdo con los objetivos del presente estudio, se realizaron dos tipos de análisis que agrupan a los objetivos específicos: el primero respecto de las percepciones docentes y de estudiantes acerca de los procesos evaluativos y un segundo, que aborda la correspondencia entre perfiles de egreso e indicadores de evaluación para los cuatro casos y las respectivas actividades curriculares seleccionadas.

\subsection{Análisis I: Percepciones docentes y de estudiantes respecto de los procesos evaluativos}

El primer análisis apuntó a recabar información acerca de la percepción de los alumnos y profesores. De este modo, el procedimiento consistió en integrar ambas para cada una de las categorías, a partir de las cuales les fueron realizadas las preguntas a los dos actores.

En cuanto a la dimensión "concepciones de la evaluación" los docentes exhiben una concepción de la evaluación como proceso, más que como un suceso momentáneo y acotado, y en la que varía la utilidad de la información recogida. Existe una tendencia a señalar que esta información es útil para la toma de decisiones por parte del docente (ocho docentes), mientras que solo tres relevan la utilidad que tiene esta información para el propio estudiante. Cabe destacar que dos de los ocho docentes que relevan la utilidad de la información para el docente, reducen la evaluación a su dimensión formal y administrativa, catalogándola incluso en un caso como un mero "requisito formal". En el caso de los estudiantes, no existe una sola percepción, aunque tanto las miradas de proceso, verificación y progreso son mayoritarias, lo cual devela percepciones más actualizadas de la evaluación, lo que dice relación tanto con los estilos evaluativos de los docentes, como con la forma en que los estudiantes la están significando. Sin embargo, cabe destacar que solo en un grupo focal se asoció la evaluación a un proceso que aporta información para la toma de decisiones. La mayor parte de los estudiantes parece ver la evaluación desde su sentido informativo y sus resultados no se relacionan con el campo de toma de decisiones en su propio proceso de aprendizaje. Sus resultados aparecen más vinculados con la utilidad que puede darle el profesor que con la utilidad que le pueden dar 
ellos mismos. Predomina, por tanto, un sentido "externalizante" de los resultados de la evaluación. Desde esta perspectiva, las percepciones respecto de los usos que se le pueden dar a los resultados de las evaluaciones son coincidentes tanto para los docentes como para los estudiantes, en tanto que no se reconoce el valor de esta información en la toma de decisiones del propio estudiante.

Lo anterior es de especial relevancia si consideramos lo planteado por McDonald, Boud, Francis y Gonczi (2000), quienes han estudiado con especial atención la forma en que la evaluación condiciona el aprendizaje, incluso mucho más que la enseñanza; señalando que se convierte en "el estímulo más significativo para el aprendizaje" (p. 45), en tanto, "todo acto de evaluación da un mensaje a los estudiantes acerca de lo que deben aprender y cómo deben hacerlo" (p. 45). El mensaje de la evaluación por lo tanto, en el caso de la muestra aquí analizada, se limitaría a señalar lo que los estudiantes deben aprender (y lo que aún no aprenden), pero no respecto de cómo hacerlo y de la toma de decisiones asociada a ello. Los autores señalados destacan que a menudo el mensaje no es explícito, no es fácilmente comprendido o es interpretado de manera diferente, recibiendo lecturas distintas por parte de los formadores.

En cuanto a la dimensión "coherencia y pertinencia" que solo fue aplicada a los docentes se consultó, en primer lugar, acerca de las variables en orden de importancia que los docentes consideran para elaborar su plan de evaluación. Aparecen aquí el programa y sus objetivos de aprendizaje, la consecución de desempeños profesionales, la aplicabilidad de lo que se va a evaluar, la pertinencia entre lo que se realiza en clases y lo evaluado. Solo un docente señala que en este ámbito considera el perfil de egreso al momento de diseñar sus evaluaciones, lo cual refleja la escasa presencia que aún poseen los perfiles al momento de diseñar y pensar las evaluaciones de las asignaturas. Esto resulta contradictorio con la información obtenida al preguntar directamente si él o ella considera el perfil de egreso de la carrera en su plan de evaluación, en cuyo caso diez de once entrevistados señalan que sí lo consideran, de manera directa o a partir de los componentes genéricos que el perfil eventualmente incluiría. Sin embargo, su inclusión se relaciona con lo que ellos 
intuyen se declara en el perfil, señalando: "Sí [incluyo el perfil en el plan de evaluación], porque yo sé que en cualquier perfil de egreso de un profesor tiene que haber una competencia vinculada con la planificación" (D2). Predomina, por tanto, la creencia de que las formas de enseñanza y evaluación o los objetivos del programa contienen implícitamente aspectos genéricos del perfil y la relación entre evaluaciones y perfil no se intenciona de manera consciente. Sin embargo, el perfil se contiene explícitamente solo en los casos donde existe un escalamiento de competencias y un trabajo con los docentes respecto de su desarrollo (caso 3).

Respecto de la dimensión "preparación de las evaluaciones", las respuestas muestran que prevalece una relación de los estudiantes con las evaluaciones de tipo tradicional, con gran predominio de la lectura y la necesidad de contar con una amplia comprensión, siendo esta la habilidad que los estudiantes privilegian con mayor nitidez. Llama la atención lo poco que se mencionan otras habilidades como la reflexión, discusión y diálogo y aplicabilidad del conocimiento, que posibilitarían otras concepciones evaluativas que son necesarias para el desarrollo de competencias y que los perfiles de egreso de las carreras intentan también promover.

En cuanto a la dimensión "usos de la evaluación y de sus resultados", todos los docentes afirman realizar retroalimentación sobre la base de los resultados de la evaluación, ya sea en la sesión en la que estos se entregan, o a través de comentarios en las evaluaciones. Sin embargo, solo uno de los once docentes señaló que la retroalimentación es puesta a disposición de los estudiantes para mejorar su trabajo y volver a presentarlo. Por supuesto, esto implica más trabajo para la docente, pues debía volver a corregir, sin embargo, el valor formativo que tiene este uso de los resultados de la evaluación es sin duda enriquecedor para el proceso, además de significar una utilización concreta y evidenciable de la retroalimentación del docente. Al respecto, Jorba y Sanmartí (2008a) señalan que es necesario que la evaluación amplíe su función meramente acreditadora o seleccionadora y reconozca dos funciones amplias; una de carácter social en términos de selección y de clasificación, así como de orientación del alumnado y otra 
de carácter pedagógico, de regulación del proceso de enseñanzaaprendizaje, es decir, "de reconocimiento de los cambios que se han de introducir progresivamente en este proceso para que todos los alumnos aprendan de forma significativa" (p. 22).

En el caso de la dimensión "metacognición de la evaluación", la totalidad de los docentes señala la existencia de oportunidades de reflexión, no obstante, esta es por lo general individual y no colectiva, ya sea con pares o con los estudiantes. También se señala predominantemente que estas instancias de reflexión son informales y que se asocian con un pensamiento personal o con una conversación con alguien. No hay ningún instrumento asociado con el proceso de reflexión acerca de la propia práctica evaluativa, solo algunas acciones de tipo parcial, como entregar uno de los instrumentos a un par o "recogiendo la información de las evaluaciones".

Dentro de esta dimensión y respecto de las propuestas de los y las estudiantes acerca de los tipos de evaluaciones que ayudarían de mejor manera al logro de las competencias declaradas, aparece de manera reiterada el reforzar el trabajo, la discusión y la retroalimentación entre los propios estudiantes. Predomina aquí una visión activa respecto de su rol en su formación y en la de sus pares que no se ha visto hasta aquí reflejada en las concepciones y prácticas evaluativas de los docentes. Sin duda lo anterior representa un enorme desafío en la formación inicial docente, dado que la integración del estudiante en los procesos evaluativos aún es externalizante y no se considera todavía su propia capacidad para autoevaluarse y coevaluar, así como su capacidad para utilizar los resultados de sus propias evaluaciones.

\subsection{Análisis II: Correspondencia entre perfiles de egreso e indicadores de evaluación para los cuatro casos y las respectivas actividades curriculares seleccionadas}

El segundo análisis, abocado a establecer la correspondencia entre perfiles de egreso e indicadores de evaluación, se realiza a partir del ingreso de competencias o habilidades del perfil de egreso relacionadas con cada asignatura de manera explícita y/o implícita, los objetivos 
o resultados de aprendizaje del programa para cada asignatura y sus correspondientes instrumentos de corrección, a una matriz de análisis con categorías predefinidas. Ello implicó el examen de las instancias evaluativas y sus respectivos instrumentos para diez asignaturas de un total de once (debido a que uno de los profesores no hizo entrega de sus instrumentos de evaluación), pudiendo tener cada una incluso hasta seis instrumentos evaluativos diferentes. Es necesario considerar que los casos 1 y 4 presentan perfiles de egreso por competencias y los casos 2 y 3 poseen perfiles por habilidades o destrezas. El perfil del caso 2 se declara como un perfil por habilidades, destrezas profesionales, áreas principales de conocimiento, actitudes y valores. Es por ello que dependiendo del caso se hablará de competencias o de habilidades (entendiendo esta última de modo amplio).

Se debe considerar de manera separada aquellas instituciones que poseen un escalamiento de las competencias o resultados de aprendizaje dentro del plan de estudios, y aquellas que solo poseen el perfil de egreso sin escalamiento, entendiendo por ello la asignación explícita que se hace de determinadas competencias a cada asignatura, dentro de un marco de progresión. En el presente estudio contamos con dos carreras que no poseen este escalamiento u operacionalización de las habilidades o competencias del perfil (caso 1 y 2) y dos que sí lo poseen (caso 3 y 4). A la asignación formal de la competencia o resultado de aprendizaje a una asignatura, le llamaremos "vinculación explícita". Para los casos sin este ordenamiento formal, se tomaron como competencias o resultados de aprendizaje relacionados aquellos que, por su nombre y ámbito, se infiere que se relacionan directamente con la asignatura, confirmándolos con las y los docentes, les llamaremos "vinculación implícita".

Como conclusión respecto de la relación entre las competencias declaradas en el perfil de egreso versus los indicadores de evaluación, en general, para ninguno de los planes de evaluación e instrumentos analizados, podríamos sostener que la correspondencia con las competencias o habilidades asociadas a cada asignatura es nula. En todos los casos hay algún nivel de correspondencia, en cuanto a los ámbitos del desempeño, los verbos y las dimensiones que aborda la competencia o habilidad y los indicadores de evaluación. 
Sin embargo, a pesar de esta correspondencia general pareciera no haber intención clara de ella en la redacción de los indicadores de evaluación. Por ejemplo, en todos los perfiles de la muestra se declara la contextualización de diversos aspectos de la enseñanza (adaptaciones curriculares, estrategias didácticas, evaluativas) a las características de los estudiantes. Sin embargo solo una asignatura de diez posee en sus instrumentos de corrección indicadores vinculados con ello. Lo anterior es coherente con las propuestas de mejora emanadas de los estudiantes en los grupos focales, orientadas a lograr una evaluación que pueda medir de mejor manera el desarrollo de competencias declaradas en el programa, en donde se señala la necesidad de contextualizar de mejor modo los planes evaluativos a las características y necesidades de los estudiantes.

Por otro lado todos los perfiles declaran el diseño, manejo o uso de métodos o estrategias de evaluación variados. Sin embargo, en las solicitudes de planificaciones, o diseños de aula, no encontramos indicadores vinculados con la demostración de esta capacidad. Si sumamos ambos aspectos, tendríamos como resultado que la contextualización del proceso de enseñanza-aprendizaje y la diversificación de las estrategias utilizadas quedan débilmente cubiertos en las asignaturas cuya responsabilidad es desarrollar estos aspectos. Tampoco es posible, sobre la base de lo anterior, asegurar que los egresados no desarrollen estos aspectos presentes en todos los perfiles. Sin embargo, el sentido de este estudio es que consideremos la importancia de la sistematización del perfil en las asignaturas, trabajando de manera conjunta con el equipo docente, para definir tanto las progresiones en su consecución, como los espacios imprescindibles en los que las asignaturas pueden desarrollar otros ámbitos de la formación, no necesariamente vinculados con este, pero resguardando el cumplimiento gradual de aquello que la institución garantiza que el futuro docente desarrollará. Mientras ello no se considere en el diseño de las programaciones de asignatura, ni se incluya en la construcción de los indicadores de evaluación, difícilmente el requisito mínimo que permite que un estudiante egrese, es decir, la aprobación de las asignaturas, representará la consecución de lo expresado en el perfil, ni las evaluaciones podrán llenarse del sentido que se espera: otorgar al docente y al estudiante información 
oportuna respecto de su proceso de aprendizaje y lineamientos para orientar el mismo hacia las metas propuestas.

La sistematización de la evaluación, a partir de la construcción de instrumentos que transparentan los criterios de evaluación a través de su expresión en indicadores y puntaje para cada uno de ellos, es un gran avance en la educación superior y representa una enorme oportunidad, así como un desafío pendiente de muchos y muchas docentes. La definición de perfiles de egreso por parte de los programas de formación profesional permite construir una carta de navegación común a un equipo de trabajo, en la que por supuesto debería esperarse que todos reciban la oportunidad de aportar, reflexionando crítica y propositivamente frente a lo que se defina en el perfil. Sin embargo, la presente investigación evidencia que no existe aún un trabajo de equipo que aborde la forma en la que efectivamente se concretizará el logro progresivo de las habilidades declaradas en el perfil dentro de cada asignatura. Las carreras que han diseñado un escalamiento de competencias, donde se explicita el ámbito en el que cada asignatura debe contribuir al logro del perfil, sin duda han dado un importante paso adelante. Sin embargo, incluso en estos casos, la incorporación de las competencias asociadas a cada asignatura en los instrumentos de evaluación, no parece un ejercicio consciente, quedando muchas veces partes importantes de aquella sin evaluarse. Si asumimos que es posible que un estudiante apruebe un programa de formación profesional de Pedagogía con un 4,0, y la universidad declara que aquellas personas que egresan cumplen el perfil de egreso, lo lógico sería por tanto que las evaluaciones y no solo los programas de las asignaturas garanticen la cobertura de las habilidades o competencias mínimas que ese estudiante debe dominar para el ejercicio de la docencia.

\section{Sugerencias generales de correspondencia}

En el informe $e^{3}$ del presente estudio se realizan diagnósticos acerca de la correspondencia entre cada instancia o grupo de instancias evaluativas

3 Disponible en http://www.cned.cl/public/Secciones/SeccionInvestigacion/investigacion_ estudios_documentos.aspx 
para cada asignatura. Sin embargo, en este apartado se sintetizan las principales recomendaciones emanadas del estudio.

A partir del análisis de 41 instrumentos de evaluación y 29 instrumentos de corrección (12 evaluaciones no poseen o no se nos hizo entrega del instrumento de corrección), correspondientes a los siete docentes que utilizan pautas de corrección en sus asignaturas, quienes facilitaron estos instrumentos para el presente estudio, es posible realizar las siguientes recomendaciones para efectivizar la correspondencia entre el perfil de egreso y los indicadores de evaluación.

a. Democratización del proceso de definición, graduación e implementación del perfil de egreso

Si bien la presente investigación no se detuvo en los procesos de definición del perfil de egreso, es posible sostener que un principio básico para la coherencia entre lo que se define y lo que se lleva a cabo es que se haga partícipe a los docentes de los procesos de consulta y construcción de los perfiles de egreso, así como de su socialización. Si se espera que los docentes inculquen actitudes democráticas en sus estudiantes, los equipos directivos y docentes debiesen adoptar funcionamientos democráticos dentro de un proceso tan importante, como es el de definir al profesional que se desea formar. Si los profesores no toman parte en este proceso, difícilmente podrán ser coherentes en sus diseños evaluativos, con algo que les resulta ajeno o impuesto.

b. Operacionalización del perfil de egreso por asignatura a través de documentos formales y socialización de los mismos

Dentro del presente estudio, el caso 4 representa un buen ejemplo de operacionalización del perfil a través de un documento. En primer lugar, este texto explicita la historia de la construcción del perfil, incluyendo la participación del consejo de carrera y cuerpo docente, además de la contratación de dos expertos en el diseño de perfiles por competencias y respectivas instancias de validación (expertos, docentes de la carrera, estudiantes de último año y 
empleadores). Luego se contextualiza el perfil en la institución en la que se enmarca, siguiendo con la facultad y por último con la carrera. Finalmente, se explicitan las cuatro dimensiones en las que se agrupan las competencias del perfil y luego se expone una tabla denominada "cruce de competencias". En ella cada competencia, según dimensión, es desagregada en resultados de aprendizaje que se correlacionan con diversas asignaturas en calidad de "tributarias" de la competencia señalada para cada apartado. Si bien el cuadro aún es muy general y aparecen, por ejemplo, 24 asignaturas vinculadas con la competencia 1.1, esta operacionalización es un intento replicable para asociar más explícitamente a las asignaturas con el perfil. Si la operacionalización del perfil es un proceso racional y sistemático y no una intuición por parte del docente, se hará más fácil la tarea de efectivizar su cumplimiento y, más importante aún, las asignaturas se verán enfrentadas a la tarea de trabajar en equipo de manera complementaria. Al respecto el desafío es cambiar nuestra atención de los procesos de enseñanza, a los proceso de aprendizaje y ello:

\begin{abstract}
“(...) nos obliga a preguntarnos si las actividades de enseñanza y aprendizaje diseñadas para cada asignatura son las más adecuadas y las más eficientes para lograr dichos aprendizajes, además de evaluar de qué forma cada resultado de aprendizaje definido, aporta al perfil de egreso de la carrera, de modo que deberemos verificar que sean pertinentes y que no estén duplicados en otras asignaturas" (Usach, 2013, p. 10).
\end{abstract}

Por último, es recomendable explicitar una progresión en la consecución de las competencias o habilidades del perfil, que permita a los docentes establecer niveles de logro, de acuerdo con la naturaleza de cada asignatura (de carácter práctica o teórica) y de acuerdo con el nivel en el que esta se encuentre (en primer o último año por ejemplo).

\title{
c. Mantener una integración diferenciada de los objetivos de la asignatura y las competencias o habilidades del perfil asociadas a ella
}

En los diez programas de asignatura revisados, seis no hacen ninguna referencia al perfil de egreso. Tres programas del mismo caso (misma institución), sí lo hacen en un apartado cuyo nombre 
es "habilidades del perfil de egreso". Ello aparece en el formato de programas de asignatura de la universidad. La última asignatura presenta exclusivamente resultados de aprendizaje extraídos del perfil de egreso de la carrera. Tenemos, por tanto, presencia de ejemplos polarizados y una tendencia a la ausencia del perfil en las programaciones de asignatura dentro de la muestra. Sin duda la ausencia total o la exclusiva presencia de las competencias o habilidades del perfil en el diseño de los cursos no podrían señalarse como lo ideal. En el primer caso, se desvincula la asignatura del diseño curricular general de la carrera del proyecto común, al que las asignaturas deben contribuir. En el segundo caso, la asignatura corre el riesgo de perder identidad, al no poseer objetivos que le sean propios y que, en definitiva, representan el énfasis que cada docente le da. Este espacio de identidad dentro de cada asignatura, asegura en cierta medida la diversidad dentro del proceso formativo, y ello sin duda es una expresión de riqueza. Así lo es también que el docente dialogue con los estudiantes las metas del curso y las trayectorias de aprendizaje para conseguirlas, pudiendo complementar o redireccionar aspectos de su programación y objetivos, sobre la base de este diálogo inicial, posibilidad que es invisible en las entrevistas. Ello también puede realizarse basándose en instancias de evaluación diagnóstica, así como consultas acerca de las expectativas del curso. En fin, las posibilidades son múltiples. Si bien sería recomendable que las asignaturas posean responsabilidades para con el perfil, es fundamental resguardar los espacios de contextualización de lo que debe enseñarse al grupo de estudiantes que se supone debe aprender. Es por ello que consideramos que el modelo del caso 3 es el que más se acerca a este equilibrio, pues en el programa se establecen tanto las habilidades del perfil, como los propósitos de la asignatura. En la asignatura A9 encontramos un muy buen ejemplo de coherencia entre las habilidades del perfil (declaradas en el programa), los propósitos de la asignatura (que complementan, profundizan y diversifican aún más lo señalado en el perfil) y los indicadores de evaluación de los instrumentos de corrección, que prácticamente le dan cobertura total a las habilidades del perfil y a los propósitos de la asignatura, confirmado esto además en la percepción de los estudiantes. 


\section{d. Sistematizar la orientación de los indicadores de evaluación a las competencias o habilidades del perfil}

A modo general podemos señalar que la principal dificultad en el logro de esta correspondencia es que no tenemos evidencia de que ella se intencione en un proceso sistemático o siquiera consciente. Sin bien los profesores la consideran en el plano declarativo, esto pareciera no explicitarse en la correspondencia entre, por ejemplo, los verbos o en aspectos específicos de la misma.

\section{Conclusiones}

El presente estudio se propuso como objetivo general diagnosticar la relación existente entre las competencias declaradas en los perfiles de egreso de diferentes carreras de Pedagogía en Educación Básica y los indicadores de evaluación utilizados en los instrumentos de corrección de tres asignaturas a lo largo del plan de estudios de estas carreras. Frente a ello podemos señalar que la principal dificultad en el logro de esta correspondencia es que no tenemos evidencia de que ella se intencione en un proceso sistemático. Sin bien los profesores la consideran en el plano declarativo, esto pareciera no explicitarse en la correspondencia entre, por ejemplo, los verbos o en aspectos específicos de la misma. Sostenemos que hay cierta correspondencia en general, pero pareciera no haber intención clara de ella en la construcción y redacción de los indicadores de evaluación.

Respecto de la hipótesis del presente estudio, y a modo general, para ninguno de planes de evaluación e instrumentos analizados podríamos sostener que la correspondencia con las competencias o habilidades asociadas a cada asignatura es nula. En todos los casos hay algún nivel de correspondencia, basada en la relación de cobertura de los indicadores de las competencias o habilidades correspondientes. Sin embargo, debido a las diferencias de coberturas, énfasis o desempeños entre las competencias y habilidades y los indicadores de evaluación, y sobre la base de las entrevistas hechas a los docentes, podríamos sostener que sea cual sea el nivel de correspondencia lograda, esta no es fruto de un ejercicio sistemático ni consciente por parte de los docentes. En el momento de diseñar y construir 
los instrumentos de corrección, la presencia de las competencias o habilidades del perfil de egreso se hace cada vez más difusa. También es preciso considerar que aún hay perfiles de egreso muy difíciles de operacionalizar, en los cuales encontramos declaraciones muy generales o amplias, en cuyos casos parece existir coincidencia entre perfil e indicadores, pues al ser expresiones tan generales, resulta más fácil que cualquier asignatura las aborde. Sin embargo, si se quiere efectivamente avanzar hacia modelos de aprendizaje por competencias o habilidades y que ello impacte de manera real en las estrategias evaluativas de cada asignatura, sería recomendable contar con perfiles más operativos en este sentido, con expresiones claras y desglosadas por área y, dentro de lo posible, con los indicadores de logro para avanzar progresivamente hacia las competencias o habilidades propuestas.

Las sugerencias de mejora, emanadas del análisis de 41 instrumentos de evaluación y 29 instrumentos de corrección, se pueden sintetizar de la manera que sigue:

Tabla 2: Recomendaciones para mejora de correspondencia entre asignaturas y sus evaluaciones, con el perfil de egreso

\begin{tabular}{l|l}
\hline Ámbito & Recomendaciones \\
\hline & $\begin{array}{l}\text { Democratización del proceso de definición, graduación e implementación del } \\
\text { perfil de egreso. }\end{array}$ \\
\cline { 2 - 2 } $\begin{array}{l}\text { Sugerencias } \\
\text { generales de } \\
\text { correspondencia } \\
\text { entre indicadores } \\
\text { y perfil de egreso }\end{array}$ & $\begin{array}{l}\text { Operacionalización del perfil de egreso por asignatura a través de documentos } \\
\text { formales y socialización de los mismos. }\end{array}$ \\
\cline { 2 - 2 } & $\begin{array}{l}\text { Sistematizar la orientación de los indicadores de evaluación a las competencias } \\
\text { o habilidades del perfil. }\end{array}$ \\
\cline { 2 - 2 } & $\begin{array}{l}\text { Sistematizar los mecanismos para que los estudiantes evalúen los instrumentos } \\
\text { de corrección que les son aplicados y realicen propuestas de mejora }\end{array}$ \\
\hline
\end{tabular}

La necesidad que este estudio pretendía develar la nueva capacidad que deben desarrollar los docentes universitarios al verse en el desafío de enmarcar sus asignaturas en un plan coherente de formación, donde sus cursos ya no constituyen un área de su 
exclusiva incumbencia y especialidad, sino que son parte de una red formativa, con un propósito que se cumple en la concreción gradual y progresiva de competencias o habilidades, y cuyas experiencias evaluativas deben, al menos, contribuir. Probablemente, los perfiles de egreso sigan cambiando, al igual que las orientaciones o regulaciones ministeriales al respecto. Sin embargo, lo que estos cambios implican para la concepción de las prácticas docentes universitarias y el funcionamiento interno de los equipos, esperamos puedan darse y permanecer para mejorar el proceso de aprendizaje tanto de los estudiantes, como de nosotros mismos como docentes.

La concepción y práctica de cada asignatura no debiese pensarse más como un elemento aislado dentro del plan de estudios, si no como parte orgánica de un proceso gradual de consecución de un objetivo, explicitado ello en un perfil de egreso. Si bien antes las carreras poseían un objetivo o un determinado perfil, su explicitación en resultados concretos y muchas veces vinculados específicamente con cada asignatura, ponen a disposición de cada profesor una nueva gama de instrumentos antes inexistentes, que permiten sentar una base para la coordinación del cuerpo docente. Por supuesto, es de esperar que tanto los perfiles como las instancias de diseño y coordinación de los mismos sean cada vez más democráticos e inclusivos, no solo con el cuerpo docente, si no con la comunidad educativa en general y con los desafíos que la misma ciudadanía vaya definiendo para la labor docente. Esperamos que instancias de socialización y desarrollo de los instrumentos de evaluación, así como el trabajo en equipo de los cuerpos docentes y la retroalimentación de este trabajo con los estudiantes, permita en el mediano o largo plazo revertir la situación planteada por Jorba y Sanmartí (2008b) cuando sostienen que "la evaluación es la práctica pedagógica que menos motiva al profesorado y que más le molesta. Al mismo tiempo, para el alumnado la evaluación es la actividad más temida y la menos gratificadora" (p. 21).

Al concluir este estudio reafirmamos lo planteado por McDonald, Boud, Francis y Gonczi (2000):

"La evaluación es el estímulo más significativo para el aprendizaje: todo acto de evaluación da un mensaje a los estudiantes acerca de lo que ellos deben aprender y cómo 
deben hacerlo. A menudo, el mensaje no es explícito, no es fácilmente comprendido o es interpretado de manera diferente, recibiendo lecturas distintas por parte de formadores y alumnos" (p. 45).

Es por ello que reiteramos como fundamental que los planes evaluativos de las asignaturas dejen de ser espacios aislados, sin correspondencia sistemática con el proyecto general de la carrera (expresado en el perfil de egreso) y a veces incluso, sin correspondencia con los objetivos de la asignatura. Frente a los desafíos que los nuevos modelos de aprendizaje suponen (ya sea por competencias, por habilidades, destrezas y actitudes, u otros) el diálogo entre currículum y evaluación debe intensificarse para resguardar la coherencia entre el discurso curricular y las prácticas evaluativas reales, de modo de centrar los esfuerzos y cambios que se realicen en este sentido en aquellos aspectos que más incidencia tienen en la orientación del aprendizaje de las y los estudiantes.

Respecto del supuesto inicial de la presente investigación, este sostuvo que las competencias o habilidades declaradas en los perfiles de egreso no se ven reflejadas de manera concreta y evidenciable en los instrumentos de evaluación y corrección de las asignaturas que componen el plan de estudios de los diversos programas, permaneciendo los perfiles aún en el plano declarativo y no en el de lo concreto y evidenciable, como por ejemplo, en las programaciones de evaluación o en los indicadores de evaluación de los instrumentos de corrección. Si bien los resultados no muestran que la congruencia sea nula o escasa, la triangulación de entrevistas, grupos focales y análisis documental permite señalar que los niveles de correspondencia logrados no son el fruto de un ejercicio sistemático o consciente de coherencia curricular y evaluativa. Por otro lado, es pertinente recalcar además la necesidad de mejoras en diferentes dimensiones y aspectos de los instrumentos de corrección, que permitirán optimizar su efectividad y su orientación con los objetivos de la asignatura o del perfil. 


\section{Referencias}

Ahumada, P. (2001). Estrategias y procedimientos para una evaluación auténtica de los aprendizajes en la enseñanza universitaria. Revista Perspectiva Educacional, (Vol. 45) 11-24.

Ahumada, P. (2006). La evaluación en una concepción de aprendizaje significativo. Valparaíso: Editorial UCV.

Ávalos, B. (2005). Competencias y desempeño profesional. Revista Pensamiento Educativo, 36, 19-32.

Ávalos, B. (2009). Formación inicial de profesores. Formación inicial docente en Chile: calidad y políticas. En C. Bellei, D. Contreras, J. Valenzuela, C. Bellei, D. Contreras y J. Valenzuela (Eds.), Ecos de la revolución pingüina: avances, debates y silencios de la reforma educacional (pp. 257284). Santiago de Chile: Universidad de Chile.

Barber, M, \& Mourshed, M. (2008). Cómo hicieron los sistemas educativos con mejor desempeño del mundo para alcanzar sus objetivos. McKinsey \& Company: PREAL. Recuperado el día 20 de abril de 2012 en http:// www.oei.es/pdfs/documento_preal41.pdf

Bardin, L. (2002). Análisis de contenido. Madrid: Ediciones Akal S.A.

Barraza, A. (2007). La formación docente bajo una conceptualización comprehensiva y un enfoque por competencias. Revista Estudios Pedagógicos XXXIII, 2, 131-153.

Boud, D. (1995). Enhancing learning through self-assessment. London: Kogan Page.

Brunner, J., Elacqua, G., Tillet, A., Bonnefoy, J., González, S., Pacheco, P. \& Salazar, F. (2005). Guiar el mercado: Informe sobre la educación superior en Chile. Santiago de Chile: Universidad Adolfo Ibáñez.

Cano, M. (2008). La evaluación por competencias en la educación superior. Revista de Currículum y Formación del Profesorado, 12(3), 1-16.

Cardelli, J. \& Duhalde, M. (2001). Formación docente en América Latina. Una perspectiva político-pedagógica. Cuadernos de Pedagogía (Vol. 308), 38-45.

Casanova, M. (2007). Manual de evaluación educativa. Madrid: Editorial la Muralla.

Centro Interuniversitario de Desarrollo, CINDA (2009). Diseño curricular basado en competencias y aseguramiento de la calidad en la educación superior. Santiago: Autor.

Comisión Nacional de Acreditación, CNA (2007). Criterios generales de evaluación para licenciaturas. Santiago: Autor. Recuperado el día 15 de 
agosto de 2014, de http://www.cnachile.cl/Documentos\%20de\%20 Paginas/Licenciaturas\%20preg.pdf

Cisternas, T. (2011). La investigación sobre formación docente en Chile. Territorios explorados e inexplorados. Revista Calidad en la Educación, 35, 131-164.

Delors, J. (1995). La educación encierra un tesoro. Informe a la Unesco de la comisión internacional sobre la educación para el siglo XXI. Madrid: Unesco.

Denzin, N. \& Lincoln, Y. (2012). El campo de la investigación cualitativa. Barcelona: Gedisa.

Gómez, H. (2013). Análisis crítico del discurso del currículum y de género de formación inicial docente de Pedagogía en Educación Básica en Chile. (Tesis de Magíster inédita). Pontificia Universidad Católica de Chile, Santiago de Chile.

Entwlstle, N. (1991). Approaches to learning and perceptions of the learning environment. Higher Education 22 (pp. 201-204), Netherlands.

González, A. (2011). Formación inicial docente basada en perspectiva de competencias profesionales. Revista Electrónica de Desarrollo de Competencias (REDEC), 2(8), 32-42.

Hagar. P; Gonczi. A. \& Athanasou. J. (1994). General issues about assessment of competence. Assessment and Evaluation in Higher Education. Vol. 9. 3-16.

Hawes, G. (2005). Evaluación de competencias en la educación superior. Proyecto Mecesup Tal 0101: Universidad de Talca.

Hawes, G. (2010). Glosario básico para la modernización curricular. Santiago de Chile: Universidad de Chile.

Jorba, J. \& Sanmartí, N. (2008a). Evaluación como ayuda al aprendizaje. Barcelona: Grao.

Jorba, J. \& Sanmartí, N. (2008b). La función pedagógica de la evaluación. En H. Quintana, M. Sbert, J. Navalón, L. Otero, N. Sanmartí, M. Fons, T. Pigrau, D. Quinquer, H. Weissman, B. Mir, M. Calatayud, I. Pitaluga, M. Ballester, M. Pujol, J. Jorba, C. Sbert, J. Batalloso, I. Moreno e I. Córdoba, Evaluación como ayuda al aprendizaje (pp. 20 30). Barcelona: Grao.

Knust, R. \& Gómez, S. (2009). La evaluación con enfoque por competencias: ¿Se implementa realmente la evaluación por competencias? Experiencias en Holanda y diferentes países de América Latina. Revista Electrónica de Desarrollo de Competencias (REDEC), 3(1), 104-125. 
Latorre, M. (2005). Continuidades y rupturas entre formación inicial y ejercicio profesional Docente. Revista Digital OEI, 37, 1-11.

Marton, F. \& Saljo, R. (1997). Approaches to learning, in F.Marton, D.Hounsell \& Entwistle, N. (Eds), The experience of learning. Implications for teaching and studying in higher education. Edinburgh: Scottish Academic Press.

McDonald, R., Boud, D., Francis, J. \& Gonczi, A. (2000). Nuevas perspectivas sobre evaluación. Unesco. Paris 1995. Boletín Cinterfor 149, mayoagosto.

Medina, M. \& Verdejo, A. (2001). Evaluación del aprendizaje estudiantil. Santo Domingo: Isla Negra Editores.

Mercado, R. (2010). El debate actual sobre la formación inicial de docentes en México. Revista Semestral da Associacao Brasileira de Psicología Escolar e Educacional, 14(1), 149-157.

Organización para la Cooperación y el Desarrollo Económicos, OCDE (2005). Teachers matter: Attracting, developing and retaining effective teachers. Paris: autor.

Organización para la Cooperación y el Desarrollo Económicos, OCDE (2013). El Aseguramiento de la calidad en la educación superior en Chile 2013. Serie Revisión de Políticas Nacionales de Educación. Santiago de Chile: Autor.

Pavié, A. (2011a). Formación docente: hacia una definición del concepto de competencia profesional docente. Revista Electrónica Universitaria de Formación del Profesorado, Vol. 14 No1, 67-80.

Pavié, A. (2011b). Enfoque basado en competencias: orientaciones sobre sus procedimiento de evaluación. Revista Electrónica de Desarrollo de Competencias (REDEC), 7(1), 114-147.

Perrenoud, P. (2001). La formación de docentes en el siglo XXI. Revista de Tecnología Educativa, 3, 503-523.

Perrenoud, P. (2004). Diez nuevas competencias para enseñar. México: SEP.

Pedraja - Rojas, L., Araneda-Guirriman, C., Rodríguez-Ponce, E., \& RodríguezPonce, J. (2012). Calidad en la formación inicial docente: evidencia empírica en las universidades chilenas. Revista Formación Universitaria, 5(4), 15-26.

Piñuel, J.L. (2002). Epistemología, metodología y técnicas del análisis de contenido. Revista de Estudios de Sociolingüistica 3(1), 2002, 1-42. Recuperado el día 20 de junio de 2014 en https://www.ucm.es/data/ cont/docs/268-2013-07-29-Pinuel_Raigada_AnalisisContenido_2002_ EstudiosSociolinguisticaUVigo.pdf. 
Ramírez, J. \& Santander, E. (2003). Instrumentos de evaluación a través de competencias. Recuperado el 21 de Septiembre de 2014 de www. pizarron.cl

Salas, W. (2009). Formación por competencias en educación superior. Una aproximación conceptual a propósito del caso colombiano. Revista Iberoamericana de Educación, 36, 1-10.

Sosa Santillán, A. (2008). Apuntes para un currículum basado en competencias. Una mirada desde Vygotski. Recuperado el 15 de agosto de 2014 de www.efis.ucr.ac.cr/varios/ponencias

Stake, R. (2007). Investigación con estudios de casos. Madrid: Morata.

Struyven, K., Dochy, F. \& Janssens, S. (2005). Students' perceptions about evaluation and assessment in higher education: a review. Assessment and Evaluation in Higher Education, 30(4), 325-341.

Tobón, S. (2006). Aspectos básicos de la formación basada en competencias. Proyecto Mecesup, Universidad de Talca.

Universidad de Santiago de Chile, Usach (2013). Manual para la implementación del sistema de créditos transferibles, SCT Chile. Santiago de Chile: Nuevamérica Impresores Ltda.

Vaillant, D. (2004). Construcción de la profesión docente en América Latina. Tendencias, temas y debates. Serie Documentos PREAL 31, 1-40.

Vaillant, D. (2009). Políticas para un desarrollo profesional docente efectivo. En D. Vaillant, y C. V. Medrano (Coord.), Aprendizaje y desarrollo profesional docente (pp. 29-39). Madrid: Santillana.

Recibido: 04/09/2014

Aceptado: 28/11/2014 